\title{
Risks associated with suspected dysphagia in infants admitted to a neonatal intensive care unit in a South African public hospital
}

\author{
J Schoeman, B Com Pathol, M Com Pathol; A Kritzinger, DPhil \\ Department of Speech-Language Pathology and Audiology, Faculty of Humanities, University of Pretoria, South Africa
}

Corresponding author: J Schoeman (jacolineschoeman2gmail.com)

\begin{abstract}
Background. The prevalence of neonatal dysphagia is increasing, as medical advances contribute to the survival of critically ill and preterm infants. Additional factors such as low birth weight (LBW), gastro-oesoephageal reflux disorder, failure-to-thrive (FTT), and HIV may increase the complexity of dysphagia symptoms. Knowledge of context-specific risk factors for dysphagia may lead to an effective pathway of diagnosis and management in vulnerable neonates.

Objective. To describe the feeding characteristics and categories of underlying medical conditions in infants of gestational age 24 - 42 weeks. Methods. The study was a retrospective review of 231 purposively selected medical and speech-language therapy records. Participants had a mean stay of 28.5 days in a neonatal intensive care unit in a peri-urban public hospital and were referred for a swallowing and feeding assessment. An existing seven-category framework for the classification of suspected dysphagia was used.

Results. Most participants (90.0\%) presented with multiple medical conditions. Underlying neurological conditions (48.5\%) and feeding difficulties secondary to systemic illness $(65.8 \%)$ contributed mostly to suspected dysphagia in the sample. It was found that $71.0 \%$ of infants presented with feeding difficulties secondary to other conditions such as LBW and prematurity, highlighting the need for an expanded dysphagia classification framework.

Conclusion. The results concur with the outcomes of previous studies and confirm the need for a unique classification framework in South Africa. Dysphagia is a complex condition and frequently cannot be attributed to a single risk factor.
\end{abstract}

S Afr J Child Health 2017;11(2):75-79. DOI:10.7196/SAJCH.2017.v11i2.1186

Dysphagia in children is ever-increasing, mostly due to the improved survival rate of infants and children with life-threatening conditions and multiple associated health problems. ${ }^{[1,2]}$ Dysphagia, a swallowing disorder secondary to a problem in one or more of the four phases of swallowing, is managed by speech-language therapists (SLTs) who are qualified to assess the dysfunction and provide intervention. ${ }^{[3-5]}$

Infants with risks such as prematurity, congenital or acquired medical conditions, or those with prolonged stays in neonatal intensive care units (NICUs) are at greater risk of developing dysphagia and nutritional problems than typically developing, healthy neonates. ${ }^{[4,6]}$ Also, infants requiring continued intervention for dysphagia are frequently those who were previously admitted to a NICU. ${ }^{[6]}$ Infants with the most complex or severe medical conditions are most at risk of presenting with disorganised or dysfunctional feeding patterns. ${ }^{[4]}$ When preterm infants present with disorganised feeding patterns, it is generally due to immaturity, whereas dysfunctional sucking patterns may be more severe and are usually associated with neurological involvement. ${ }^{[4]}$ It therefore appears that a close relationship exists between dysphagia, the infant's medical diagnosis, associated conditions, and the severity thereof.

Limited research regarding risk factors associated with dysphagia in infants admitted to the NICU is currently available. ${ }^{[4,7,8]}$ This is a concern in developing countries, where the burden of disease is high. ${ }^{[9]}$ In a developing country such as South Africa (SA), numerous additional challenges, such as the effects of poverty and HIV, may contribute to dysphagia in infants. ${ }^{[9]}$ HIV may affect all phases of swallowing as a result of oral thrush, odynophagia and gastro-oesophageal reflux disorder (GERD), which can lead to failure-to-thrive (FTT). ${ }^{[10]}$ The prevalence of dysphagia and
GERD among children with HIV is poorly recorded, but frequently encountered in clinical practice and may contribute significantly to the morbidity of infants with exposure to the virus. ${ }^{[10]}$ The incidence of dysphagia in typically developing children is estimated at 25 $45 \%$, and even higher, up to $80 \%$, in children with developmental disabilities. ${ }^{[1]}$ The prevalence of neonatal dysphagia is unknown, but represents a universal problem, as dysphagia may carry over to infancy and toddler-age groups. ${ }^{[12]}$

It is important to identify dysphagia as soon as possible after birth, while the infant is still in the hospital, so that the appropriate short- and long-term dietetic and SLT management and parent training can commence. ${ }^{[4]}$ SLTs should be able to state when an infant is not ready for oral feeding and maximise oral feeding skills and safety in those infants who are ready to feed orally. ${ }^{[1,5]}$ Left unidentified and untreated, dysphagia can lead to FTT, GERD, aspiration pneumonia and an inability to establish and sustain vital nutrition and hydration. ${ }^{[13]}$

The objectives of the study were to describe the feeding characteristics of infants admitted to a NICU and referred for suspected dysphagia in a public hospital, and to determine which medical conditions were associated with the participants. Identifying risk factors can contribute to a better understanding of infants with suspected dysphagia, which may lead to improved referral guidelines and SLT staff-planning to ensure adequate intervention for all. A holistic understanding of the diversity of context-specific risk factors, associated with suspected dysphagia in infants who are already compromised by medical conditions, may be attained.

\section{Methods}

The study was a retrospective review of medical and SLT records from 2010 to 2014 and included infants, aged 24 - 42 weeks' gestational age 
(GA) at birth, who were admitted to a peri-urban public hospital. GA was determined using the mother's last menstrual period. Birth weight was defined as: normal (>2 $500 \mathrm{~g}$ ); low birth weight (LBW; $<2500 \mathrm{~g})$, very LBW $(<1500 \mathrm{~g})$ and extremely LBW $(<1000 \mathrm{~g})$. All participants were referred by medical doctors, nurses, audiologists, SLTs, and dieticians for a clinical swallowing evaluation.

\section{Participants}

A total of 312 infants were referred for dysphagia assessments within the study period, of which 231 complete data sets were available. Inclusion criteria were that the infants had to present with symptoms of dysphagia, be referred for a feeding or swallowing evaluation by a healthcare professional, admitted to the NICU and assessed for dysphagia by a SLT. Common symptoms of dysphagia in the participants include oral phase symptoms such as absent oral reflexes, absent or poor primitive reflexes, weak suck, uncoordinated suck, immature biting, poor bolus propulsion and poor bolus containment. Abnormalities in triggering of the swallow include absent swallow, delayed trigger of swallow, suck-swallow-breathing (SSB) incoordination, and pharyngeal phase symptoms include laryngeal penetration, aspiration, choking, pharyngeal residue and nasopharyngeal reflux. ${ }^{[14]}$

Common criteria by healthcare professionals for referral of infants and children for feeding and swallowing evaluation included: suckling and swallowing incoordination, weak suck, breathing disruptions or apnoea during feeding, excessive gagging or recurrent coughing during feeds, diagnosis of disorders associated with dysphagia or under-nutrition, severe irritability during feeding, history of recurrent pneumonia and feeding difficulty, concern for possible aspiration during feeds, lethargy or decreased arousal during feeds, tedious feeding times and nasopharyngeal reflux during feeding.

\section{Materials}

The materials used during data collection included the Neonatal Patient Discharge Report, which is available in electronic format from the local NICU database, and the SLT records, including a dysphagia assessment form. ${ }^{[15-17]}$ Data were independently collected over five years by seven different SLTs who were trained to use the same data collection instrument. A classification framework described by Arvedson and Brodsky ${ }^{[1]}$ to determine the aetiology or risk factors of paediatric dysphagia was used to categorise each participant. These categories included conditions with neurological involvement (such as asphyxia and convulsions), anatomical and structural impairments (including laryngomalacia), genetic and chromosomal disorders (including trisomy 21), dysphagia secondary to systemic illness (including pneumonia), psychosocial factors (including oral deprivation) as well as dysphagia secondary to resolved medical conditions (including hospital-acquired infections). In a local study, Fourie $^{[7]}$ expanded on the framework by adding a seventh category, 'other', as the prevalence of prematurity, LBW, GERD, and FTT is high in SA, and does not fit within any of the other six categories. ${ }^{[7]}$

\section{Procedures}

The Research Ethics Committees of two different universities granted approval for the study. Data were manually captured from the printed records to an Excel spreadsheet and analysed using SPSS version 22 (IBM Corp., USA). Being a retrospective study, there was no direct contact with mothers or infants. Variables included those in Arvedson and Brodsky's ${ }^{[1]}$ framework for categorisation of risks for dysphagia, which was expanded by Fourie ${ }^{[7]}$ to include prenatal risks (such as age of mother, number of antenatal visits), perinatal risks (such as type of delivery, Apgar scores), and postnatal medical risks (such as enteral and parenteral feeding). Pivot tables were used to determine the distribution of each participant within the different categories of medical conditions associated with suspected dysphagia. Standard deviations were calculated. Descriptive statistics were used to identify the feeding characteristics and risk factors.

Table 1. Participant characteristics $(N=231)$

\begin{tabular}{ll}
\hline Characteristic & $n(\%)^{\star}$ \\
\hline Nationality of mother & \\
South African & $204(88.3)$ \\
Non-South African & $27(11.7)$ \\
Gender & \\
Male & $111(48.1)$ \\
Female & $120(51.9)$ \\
GA at birth (weeks), mean (SD) & $34.9(3.9)$ \\
Birth weight & \\
Normal & $105(45.5)$ \\
LBW & $82(35.5)$ \\
Very LBW & $38(16.4)$ \\
Extremely LBW & $6(2.6)$ \\
Time spent in NICU (days), mean (SD) & $28.5(36.9)$ \\
HIV status of mother (N=196) & \\
Negative & $135(69.6)$ \\
Positive & $59(30.4)$ \\
GA = gestational age; SD = standard deviation; LBW = low birth weight; \\
NICU = neonatal intensive care unit. \\
*Unless otherwise specified.
\end{tabular}

\section{Results}

\section{Participant description}

The participant characteristics are described in Table 1. Most mothers were SA citizens. There were slightly more female (51.9\%) than male (48.1\%) participants, and the mean (standard deviation (SD)) GA of participants was 34.9 (3.9) weeks. The participants were mostly late preterm. More than half of the participants $(54.5 \%)$ were LBW, very LBW or extremely LBW (Table 1). The mean (SD) stay in the NICU was 28.5 (36.9) days. The percentage of mothers who were HIV-positive and, by implication, had infants exposed to the virus, corresponds with the 2012 antenatal sentinel HIV prevalence survey. ${ }^{[18]}$ The estimated HIV prevalence in the survey was $29.5 \%$ in pregnant women and in this study, $30.4 \%$ of mothers were HIV-positive. ${ }^{[18]}$ It is not known how many of the mothers were receiving antiretroviral treatment.

\section{Feeding characteristics of participants}

Table 2 shows the feeding characteristics of the participants. All participants presented with one or more symptoms of dysphagia. This can explain the frequency of parenteral (14.4\%) and enteral feeding (65.0\%), as infants with dysphagia often require alternative feeding methods to obtain adequate nutrients and fluids. ${ }^{[1]}$ Thirty-six (15.6\%) participants presented with severe feeding difficulties or signs of aspiration. An instrumental assessment, a video fluoroscopic swallow study (VFSS) was only conducted in $14(38.8 \%)$ of those participants. Instrumental assessments are recommended if there are concerns about risks for aspiration, safety of the airway, or possibilities of GERD. ${ }^{[1]}$ The reasons why VFSS was not conducted in the 22 remaining participants referred for the procedure included: participants demised before VFSS could be conducted $(n=7)$; VFSS screening machine was not functioning $(n=1)$; participant was ventilated $(n=1)$; participant was lethargic $(n=1)$; unstable or desaturating during feeding $(n=3)$; clinically 
Table 2. Feeding characteristics of participants $(N=231)$

\begin{tabular}{ll}
\hline Characteristic & $n(\%)$ \\
\hline Previous parenteral feeding & $33(14.4)$ \\
Yes & $198(85.6)$ \\
No & \\
Previous enteral (NGT/OGT) feeding & $150(65.0)$ \\
Yes & $61(26.4)$ \\
No & $20(8.6)$ \\
Unknown & \\
Referred for VFSS by doctors and SLTs $(N=36)$ & $6(15.6)$ \\
Yes & $30(84.4)$ \\
No & \\
VFSS conducted (in referrals) & $14(38.8)$ \\
Yes & $22(61.2)$ \\
No & \\
Manner of feeding at discharge & $127(55.1)$ \\
Mixed & $69(29.7)$ \\
Exclusive breastfeeding & $14(6.1)$ \\
Gastrostomy & $12(5.2)$ \\
Exclusive bottlefeeding & $6(2.6)$ \\
Cup & $3(1.3)$ \\
Syringe & \\
NGT = nasogastric tube; OGT = orogastric tube; VFSS = video fluoroscopic \\
swallow study; SLTs = speech-language therapists.
\end{tabular}

aspirating but no suck/swallow palpable $(n=4)$, clinical swallow present even though there were risks for aspiration $(n=5)$. More than half of participants (55\%) used a mixed manner of feeding such as breast- and cupfeeding, or cup- and syringe feeding. Only $29.7 \%$ of participants could breastfeed exclusively, which was related to preterm birth and LBW in most of the participants. The use of mixed feeding methods among the participants may indicate that the infants experienced breastfeeding difficulties, as establishing successful breastfeeding may be a challenge for many preterm infants and their mothers, owing to neonatal feeding difficulties. ${ }^{[19]}$ These difficulties may be due to incoordination of SSB as the suckling patterns of preterm infants often remain significantly less efficient than those of full-term infants at term age and beyond. ${ }^{[2,19]}$

Furthermore, $6.1 \%$ of the participants required long-term tube feeding, such as a gastrostomy. In another study conducted in SA, it was found that infants and children requiring gastrostomies were likely to present with multiple diagnoses, of which neurological and/ or gastrointestinal impairments were the most prominent medical conditions. ${ }^{[2]}$

\section{Underlying medical conditions in participants}

Underlying medical conditions in participants were classified according to the framework by Arvedson and Brodsky, ${ }^{[1]}$ expanded by Fourie, ${ }^{[7]}$ to determine the aetiology or risk factors of dysphagia (Table 3). Since most participants were not classified in a single category, and presented with multiple risks, the total in Table 3 does not add to $100 \%$.

The majority of the infants (71.0\%) presented with conditions that were not included in the risks of dysphagia described by Arvedson and Brodsky, ${ }^{[1]}$ a classification system developed for conditions in a developed country such as the USA. The risks included FTT,
Table 3. Underlying medical conditions in participants

\begin{tabular}{lll}
\hline Category & Description & $n(\%)$ \\
\hline A & Neurological conditions & $112(48.5)$ \\
B & Anatomical and structural conditions & $19(8.2)$ \\
C & Secondary to systemic illness & $152(65.8)$ \\
D & Chromosomal (genetic) conditions & $18(7.8)$ \\
E & Psychosocial conditions & $4(1.7)$ \\
F & Secondary to resolved medical condition & $32(13.9)$ \\
G & Other (FTT, LBW, prematurity) & $164(71.0)$ \\
FTT = failure-to-thrive; LBW = low birth weight. &
\end{tabular}

GERD, LBW as well as HIV exposure. It was found that $65.8 \%$ of participants had feeding difficulties secondary to a systemic illness, such as respiratory distress syndrome, cardiac abnormalities, and pneumonia. This could be due to the fact that preterm infants with LBW are more at risk of developing systemic illnesses, ${ }^{[13]}$ and more so in a developing country, such as SA. ${ }^{7]}$ Results indicated that $48.5 \%$ of participants had a condition with neurological involvement, such as asphyxia. The literature suggests that infants with neurological conditions, birth trauma, as well as pre- and perinatal asphyxia, are commonly found to have feeding difficulties. ${ }^{[1]}$

The conditions that occurred the least in the participants were feeding difficulties secondary to resolved medical conditions (13.9\%), including iatrogenic conditions such as hospital-acquired infections. A total of $8.2 \%$ of the participants presented with anatomical or structural conditions, such as cleft lip and palate, laryngomalacia and tracheo-oesophageal fistula, while only $7.8 \%$ of the 231 participants presented with genetic or chromosomal abnormalities, which included infants with trisomies 13,18 and 21, and other syndromes. Only $1.7 \%$ of the participants presented with psychosocial conditions such as oral deprivation and under-nutrition due to social problems. When analysing the results, it became clear that a true profile of multiple underlying conditions to feeding difficulties in the participants could not have been obtained if single categories of risk were considered.

\section{Combinations of risk conditions associated with suspected dysphagia}

Combinations of risk categories in participants are described in Table 4. The results indicate that $90.0 \%$ of participants presented with multiple medical conditions, therefore revealing the complexity of combinations of different categories. A total of 36 different combinations were found, ranging from a single category to five different combinations. Most of the participants presented with two $(50.2 \%)$ or three $(28.1 \%)$ categories of risk factors and a total of $11.7 \%$ participants presented with four or five categories of risks. The minority (10.0\%) of participants presented with a single category of risk for dysphagia. The results display the diversity and complexity of medical conditions in infants with symptoms of dysphagia. The results are in agreement with Jadcherla, ${ }^{[12]}$ who states that neonatal dysphagia can rarely be associated with a single aetiology.

\section{Discussion}

Dysphagia symptoms were accompanied by multiple medical conditions in most of the participants. As was found in other local studies, ${ }^{[7,20]}$ participants presented with a great variety of medical conditions and combinations of these conditions that either directly or indirectly affected their feeding ability. ${ }^{[7]}$

The high number of participants with neurological conditions in this sample can be explained by the fact that infants with neurological 
Table 4. Combinations of risks for dysphagia $(N=231)$

\begin{tabular}{|c|c|}
\hline Category & $n(\%)$ \\
\hline Single risk category & $23(10.0)$ \\
\hline Neurological & $6(2.6)$ \\
\hline Anatomical & $2(0.9)$ \\
\hline Dysphagia SSI & $10(4.3)$ \\
\hline Other & $5(2.2)$ \\
\hline Two risk categories & $116(50.2)$ \\
\hline Anatomical, genetic & $1(0.4)$ \\
\hline Neurological, SSI & $33(14.3)$ \\
\hline Neurological, other & $7(3.0)$ \\
\hline SSI, other & $65(28.1)$ \\
\hline Anatomical, SSI & $4(1.7)$ \\
\hline SSI, SRMC & $1(0.4)$ \\
\hline SSI, neurological & $1(0.4)$ \\
\hline SSI, genetic & $4(1.7)$ \\
\hline Three categories & $65(28.1)$ \\
\hline Neurological, SSI, other & $39(16.9)$ \\
\hline Neurological, SRMC, other & $1(0.4)$ \\
\hline Anatomical, SSI, other & $4(1.7)$ \\
\hline SSI, SRMC, other & $10(4.3)$ \\
\hline SSI, genetic, other & $2(0.9)$ \\
\hline Neurological, SSI, SRMC & $4(1.7)$ \\
\hline SSI, psychosocial, SRMC & $1(0.4)$ \\
\hline Neurological, anatomical, SSI & $1(0.4)$ \\
\hline SSI, psychosocial, other & $1(0.4)$ \\
\hline Neurological, other, SSI & 1. $(0.4)$ \\
\hline Neurological, SSI, genetic & $1(0.4)$ \\
\hline Four risk categories & $22(9.5)$ \\
\hline Anatomical, SSI, genetic, other & $3(1.3)$ \\
\hline Neurological, SSI, SRMC, other & $9(3.9)$ \\
\hline Anatomical, SSI, SRMC, other & $1(0.4)$ \\
\hline Neurological, anatomical, SSI, other & $3(1.3)$ \\
\hline SSI, genetic, SRMC, other & $1(0.4)$ \\
\hline Neurological, SSI, psychosocial, other & $1(0.4)$ \\
\hline Neurological, Genetic, SSI, Other & $1(0.4)$ \\
\hline SSI, Psychosocial, SRMC, Other & $1(0.4)$ \\
\hline Neurological, SSI, Genetic, Other & $2(0.9)$ \\
\hline Five risk categories & $5(2.2)$ \\
\hline Neurological SSI, Genetic, SRMC, Other & $1(0.4)$ \\
\hline Anatomical, SSI, Genetic, SRMC, Other & $1(0.4)$ \\
\hline Neurological, Anatomical, SSI, Psychosocial, Other & $2(0.9)$ \\
\hline Neurological, Anatomical, SSI, Psychosocial, Other & $1(0.4)$ \\
\hline
\end{tabular}

conditions are commonly found to have feeding difficulties. ${ }^{[1,13]}$ It is estimated that $85-90 \%$ of infants and children with neurological conditions, such as cerebral palsy, will present with dysphagia at some point in their lives. ${ }^{[1]}$ Further, the high incidence of systemic illnesses, such as pneumonia, in paediatric populations with dysphagia is linked to specific diagnoses, such as trisomy 21 , asthma, GERD, lower respiratory tract infection, and moist cough. ${ }^{[14]}$ Literature indicated that paediatric patients with multisystem diagnoses, in addition to dysphagia, appear to be at greatest risk for developing pneumonia. ${ }^{[14]}$ It is therefore evident that infants can present with multiple variations of swallowing impairments, such as those found in the participants of this study. ${ }^{[14]}$

The results indicate that the seven-category framework used for classification of risks for dysphagia in participants was successful to describe the complexities of different risk categories that may underlie neonatal dysphagia. Fourie ${ }^{[7]}$ found that $52 \%$ of participants had aetiological factors for dysphagia pertaining to the 'other' category. ${ }^{[7]}$ In the current study, the high rate of $71.0 \%$ participants in the 'other' category included those with HIV exposure, as there was no dedicated category for infants exposed to HIV. Therefore, the results indicated a need for an expanded classification system and the importance of an additional risk category was highlighted. It is proposed that the framework as described by Arvedson and Brodsky should be expanded to an eight-category classification framework that includes a category for prematurity, LBW and related conditions (described by Fourie ${ }^{[7]}$ as 'other') as well as a category for infants exposed to HIV. HIV exposure in infants is associated with preterm birth. ${ }^{[21]}$ As a result of prematurity and LBW, the infant is at risk for dysphagia after birth ${ }^{[19]}$ and when HIV infection becomes apparent, feeding and swallowing can be affected due to encephalopathy. An additional category would provide information regarding feeding characteristics, and aid in early identification of dysphagia.

\section{Conclusion}

Dysphagia frequently occurs in infants and is highly complex in nature. ${ }^{[12]}$ Within the context of a developing country, classifying dysphagia can be challenging and therefore an expanded framework may be beneficial. The eight-category framework can be used by healthcare personnel to refer infants for dysphagia assessment and intervention, and can be used by SLTs to identify infants at risk for dysphagia. Being a retrospective study, various limitations were present, including missing data as well as the restricted geographical location. The outcomes of the current study correspond with international research describing several risk factors for dysphagia related to the primary medical diagnosis and its sequelae, and may be present throughout the infants' hospitalisation. ${ }^{[8,12]}$ Due to the increased survival rate of preterm infants and infants with complex medical conditions, it is suggested that more research regarding neonatal dysphagia in developing countries should be conducted.

Acknowledgements. None.

Author contributions. JS was the researcher and prepared the manuscript. AK co-authored the manuscript and supervised the research. Funding. None.

Conflicts of interest. None.

1. Arvedson JC, Brodsky L. Pediatric swallowing and feeding: Assessment and management. 2nd ed. New York: Delmar Cengage Learning, 2002.

2. Kakodkar K, Schroeder JW. Pediatric dysphagia. Pediatr Clin N Am 2013;60:969-977. https://doi.org/10.1016/j.pcl.2013.04.010

3. Bell H, Sheckman Alper MA. Assessment and intervention for dysphagia in infants and children: Beyond the neonatal intensive care unit. Semin Speech Lang 2007;28(3):213-222. http://doi.org/10.1055/s-2007-984727

4. Hawdon JM, Beauregard N, Slattery J, Kennedy G. Identification of neonates at risk of developing feeding problems in infancy. Dev Med Child Neurol 2000;42(4):235-239. https://doi.org/10.1055/s-2007-984727 
5. American Speech-Language-Hearing Association. Roles of speech-language pathologists in swallowing and feeding disorders: Technical report. www.asha. org/policy (accessed 8 August 2015).

6. Sundseth Ross E, Browne V. Developmental progression of feeding skills: An approach to supporting feeding in preterm infants. Semin Neonatol 2002; 7:469-475. https://doi.org/10.1055/s-2007-984727

7. Fourie A. The aetiology and nature of paediatric dysphagia ( 0 - 18 months) in state hospitals [dissertation]. Johannesburg: University of the Witwatersrand; 2011.

8. Rommel N, De Meyer AM, Feenstra L, Veereman-Wauters G. The complexity of feeding problems in 700 infants and young children presenting to a tertiary care institution. J Pediatr Gastroenterol Nutr 2003;37(1):75-84. https://doi. org/10.1097/00005176-200307000-00014

9. Olusanya BO, Ruben RJ, Parving A. Reducing the burden of communication disorders in the developing world. An opportunity for the Millennium Development Project. J Am Med Assoc. 2006;296(4):441-444. https://doi. org/10.1001/jama.296.4.441

10. Rabie H, Marais BJ, van Toorn R, et al. Important HIV-associated conditions in HIV-infected infants and children. SA Fam Pract 2007;49(4)19-23. https://doi. org/10.1080/20786204.2007.10873538

11. Arvedson JC. Assessment of pediatric dysphagia and feeding disorders: Clinical and instrumental approaches. Dev Disabil Res Rev 2008;14:118-127. https:// doi.org/10.1002/ddrr.17
12. Jadcherla S. Dysphagia in the high-risk infant: Potential factors and mechanisms. Am J Clin Nutr 2016;20:1-7. https://doi.org/10.3945/ajcn.115.110106

13. Prasse JE, Kikano GE. An overview of pediatric dysphagia. Clin Pediatr 2009;48(3):247-251. https://doi.org/10.1177/0009922808327323

14. Dodrill P, Gosa MM. Paediatric dysphagia: Physiology, assessment, and management. Ann Nutr Metab 2015;66(5):24-31. https://doi.org/10.1159/000381372

15. McGrath JM. Feeding. In: Kenner C, McGrath JM, eds. Developmental Care of Newborns \& Infants: A Guide for Professionals. USA: Mosby, 2004:321-342.

16. Hall KD. Paediatric Dysphagia Resource Guide. Canada: Singular-Thomson Learning, 2001.

17. Fraker C, Walbert L. From NICU to Childhood: Evaluation and Treatment of Pediatric Feeding Disorders. Austin: PRO-ED, 2003.

18. National Department of Health, 2013. The 2012 National antenatal sentinel HIV and Herpes simplex type-2 prevalence survey in South Africa, 2012. https://www.health-e.org.za/wp-content/uploads/2014/05/ASHIVHerp_ Report2014_22May2014.pdf (accessed 16 February 2016).

19. Dodrill P. Feeding difficulties in preterm infants. ICAN 2011;3(6):324-331. https://doi.org/10.1177/1941406411421003

20. Norman V, Singh SA, Hittler T, et al. Indications, medical conditions and services related to gastrostomy placement in infants and children at a tertiary hospital in South Africa. S Afr J Child Health 2011;5(3):86-89.

21. Short CS, Taylor GP. Antiretroviral therapy and preterm birth in HIV-infected women. Expert Rev Anti Infect Ther 2014;12(3):293-306. http://doi.org/10.158 $6 / 14787210.2014 .885837$ 